\title{
Template-free Synthesis of Hierarchically Porous SiBCN Monolith from Organic Gel Precursors
}

Lujiao Yang, ${ }^{,+}$Jianhua Zou, ${ }^{+}$Ying Zhang, ${ }^{\dagger}$ Gang Shao, ${ }^{+}$Changan Wang, ${ }^{\xi, *}$ and Linan $A n^{t, *}$

'Department of Materials Science and Engineering, Xiamen University, Xiamen, Fujian 361005, China

${ }^{\ddagger}$ Advanced Materials Processing and Analysis Center, University of Central Florida, Orlando, FL 32816, USA

${ }^{\S}$ Department of Materials Science and Engineering, Tsinghua University, Beijing 100084, China

Abstract: A novel template-free method to synthesize hierarchically porous $\mathrm{SiBCN}$ monolith from a tailor-made organic gel precursor is proposed. The process includes four steps: the condensation reaction of tris[dichloromethylsilylethyl]borane and bis(trimethylsilyl)carbodiimide, the replacement of byproduct trimethylchlorosilane by styrene solution, the polymerization of styrene and the pyrolysis at $1000{ }^{\circ} \mathrm{C}$. The structures and thermal properties of organic gel precursors and $\mathrm{SiBCN}$ monolith, as well as the high temperature properties of $\mathrm{SiBCN}$ monolith were studied. This work demonstrates that the introduction of polystyrene is the key to obtain stable three-dimensional porous $\mathrm{SiBCN}$ network. The obtained $\mathrm{SiBCN}$ monolith displays a 
hierarchically porous structure with an average pore size of $1.95 \mathrm{~nm}$, a pore volume of $0.335 \mathrm{~cm}^{3} \cdot \mathrm{g}^{-1}$ and a specific surface area of $31.78 \mathrm{~m}^{2} \cdot \mathrm{g}^{-1}$. It is also observed that such hierarchically porous structure could be maintained after annealing at $1500{ }^{\circ} \mathrm{C}$. A pore size homogenization process is proposed to clarify the changes of porous architectures during the high temperature annealing.

Keywords: Hierarchically porous; Polymer derived ceramics; SiBCN; Template-free

\section{Introduction}

Hierarchically porous ceramics (HPCs) are a kind of ceramics containing macropores $(>50 \mathrm{~nm})$, mesopores $(2 \sim 50 \mathrm{~nm})$ and/or micropores $(<2 \mathrm{~nm})[1]$. Due to the special hierarchical architecture, HPCs possess high surface area and large pore volume combining with excellent interconnected mass transport channels [1]. With these characteristic properties, HPCs are much more effective for applications in drug delivery, catalysis, energy conversion, photonic devices, gas storage, sensors, and filtration/separation than single-size porous counterparts [1-5]. Previous studies in this field were primarily focused on porous oxide ceramics. However, these oxide ceramics are not suitable for applications such as high temperature sensors due to their limited thermostructural and thermochemical stability [6-9]. Consequently, research efforts have been made to obtain hierarchically porous non-oxide ceramics which exhibit better thermal stability and chemical inertness $[4,5,10-13]$. A variety of synthesis methods 
such as replica, sacrificial template, direct foaming, and freeze-drying have been explored [13-17]. One of the most promising non-oxide ceramics is polymer-derived silicoboron carbonitride $(\mathrm{SiBCN})$ ceramic, which exhibits excellent stability at ultra-high temperature up to $2000{ }^{\circ} \mathrm{C}$ [8, 18-28]. In addition, compared to polycrystalline ceramics prepared by powder metallurgy based techniques, polymer-derived ceramics (PDCs) exhibit a set of unique properties such as high-temperature semiconducting and piezoresistivity [29-32]. The direct chemical-to-ceramic process of PDCs also offers an unusual flexibility to synthesize components with various shapes and structures [33-38]. Majoulet and coworkers reported the synthesis of hierarchically porous $\mathrm{SiBCN}$ ceramic monolith by spark plasma sintering (SPS) of ordered mesoporous SiBCN powders [4]. Nevertheless, synthesis of hierarchically porous $\mathrm{SiBCN}$ ceramics has not been extensively explored.

Herein, we report a new template-free method to synthesize a hierarchically porous SiBCN monolith from a tailor-made organic gel precursor. The structures and thermal properties of the organic gel precursors and the obtained porous SiBCN monolith were studied in detail. With the good formability of the organic gel precursors, this method could be further used to in-situ prepare various hierarchically porous non-oxide ceramic monoliths in different shapes.

\section{Experimental section}




\section{1}

\section{bis(trimethylsilyl)carbodiimide}

2.1.1 Tris[dichloromethylsilylethyl]borane (TCB). TCB is a common monomer to synthesize boron-containing polymer precursors of SiBCN ceramics [21, 26]. The unique feature of $\mathrm{TCB}$ is the $\mathrm{Si}-\mathrm{Cl}$ bonds, which facilitate the formation of a three-dimensional (3D) network structure. First, $11.8 \mathrm{ml}$ borane-dimethylsulfide complex $\left[\mathrm{BH}_{3} \cdot \mathrm{S}\left(\mathrm{CH}_{3}\right)_{2}\right](2 \mathrm{M}$ in toluene solution) was added dropwisely into $10 \mathrm{~g}$ dichloromethylvinylsilane $\left(\mathrm{C}_{3} \mathrm{H}_{6} \mathrm{Cl}_{2} \mathrm{Si}\right)$ at $0{ }^{\circ} \mathrm{C}$. The mixture was then stirred at room temperature (RT) for $24 \mathrm{~h}$ to allow the hydroboration reaction between $\left[\mathrm{BH}_{3} \cdot \mathrm{S}\left(\mathrm{CH}_{3}\right)_{2}\right]$ and $\mathrm{C}_{3} \mathrm{H}_{6} \mathrm{Cl}_{2} \mathrm{Si}$. In the end, oily transparent TCB was obtained by vacuum drying to remove volatile components such as toluene and dimethylsulfide.

2.1.2 Bis(trimethylsilyl)carbodiimide (BTSC). First, $16.8 \mathrm{~g}$ dicyanamide, $70.8 \mathrm{~g}$ hexamethyldisilazane and $0.1 \mathrm{~g}$ ammonium sulfate were mixed in a $250 \mathrm{ml}$ flask with a reflux condenser. The temperature was then gradually increased to $160{ }^{\circ} \mathrm{C}$. After $3 \mathrm{~h}$ reaction, the mixture was turned into a transparent liquid. BTSC was then collected by a fractional distillation of the transparent liquid at $162^{\circ} \mathrm{C}$.

\subsection{Synthesis of organic gel precursors}

The obtained TCB and BTSC were used to synthesize organic gel precursor for the hierarchically porous $\mathrm{SiBCN}$ ceramic monolith. First, $1.50 \mathrm{~g}$ BTSC and $1.17 \mathrm{~g}$ TCB were mixed together at $60{ }^{\circ} \mathrm{C}$ for $18 \mathrm{~h}$ in a cylindrical glass vial to form a light yellow 
soft boron-modified polysilylcarbodiimide (BPSC) wet gel. Liquid trimethylchlorosilane $\left(\mathrm{Me}_{3} \mathrm{SiCl}\right)$ was generated as a byproduct. The BPSC wet gel was then immersed in a styrene solution (with $1 w t \%$ AIBN (azodiisobutyronitrile) as the initiator) for $4 \mathrm{~h}$ and consequently washed with the styrene solution. The gel was repeatedly immersed and washed to remove $\mathrm{Me}_{3} \mathrm{SiCl}$ completely. Additional heating at $80{ }^{\circ} \mathrm{C}$ for $4 \mathrm{~h}$ of the gel was then applied to polymerize styrene to solid polystyrene (PS). As a result, a yellow hard organic xerogel precursor monolith with a cylinder shape was obtained and named as BPSC-PS.

\subsection{Synthesis of porous SiBCN Ceramic Monolith}

The BPSC-PS monolith precursor was first heated at $400{ }^{\circ} \mathrm{C}$ for $4 \mathrm{~h}$, named BPSC-PS-400. BPSC-PS-400 was then pyrolyzed at $1000{ }^{\circ} \mathrm{C}$ for $4 \mathrm{~h}$ under a nitrogen flow to obtain porous SiBCN ceramic monolith (named SiBCN-1000). The ceramic yield was $15 \%$. SiBCN-1000 was further annealed at $1500{ }^{\circ} \mathrm{C}$ for $4 \mathrm{~h}$ under a nitrogen flow and the obtained product is named SiBCN-1500.

\subsection{Characterization}

Fourier transform infrared (FT-IR) spectra of TCB, BTSC, BPSC-PS and BPSC-PS-400 were measured with a Perkin Elmer 100 spectrometer. The FT-IR spectrum of SiBCN-1000 was measured on a Nicolet Avatar 360 in a $\mathrm{KBr}$ pellet. ${ }^{1} \mathrm{H}$ NMR, ${ }^{29} \mathrm{Si}\left\{{ }^{1} \mathrm{H}\right\}$ CP MAS NMR and ${ }^{11} \mathrm{~B}\left\{{ }^{1} \mathrm{H}\right\}$ CP MAS NMR were measured on a 
Bruker AV300 spectrometer. The solvent for ${ }^{1} \mathrm{H}$ NMR was $\mathrm{CDCl}_{3}$. The thermogravimetric analysis (TGA) curves were obtained using a TA Q5000 instrument with a heating rate of $20^{\circ} \mathrm{C} / \mathrm{min}$ up to $950{ }^{\circ} \mathrm{C}$ under a nitrogen flow. Scanning electron microscope (SEM) images were obtained using a Zeiss Ultra-55 field emission scanning electron microscope (FESEM). Nitrogen adsorption/desorption isotherms were measured at $77 \mathrm{~K}$ in a nitrogen atmosphere using Autosorb iQ Station 2. The density of SiBCN-1000 was measured with Archimedes' method using water as the immersion medium. X-ray diffraction (XRD) measurements were carried out on a Rigaku Ultima IV X-ray diffractometer using a $\mathrm{Cu} K_{\alpha}$ radiation with a wavelength of $0.154 \mathrm{~nm}$.

\section{Results and discussion}

\subsection{Structures and pyrolysis properties of organic gel precursors}

The key procedures to obtain 3D porous $\mathrm{SiBCN}$ ceramic monolith are the synthesis of organic gel precursor BPSC and keeping its 3D network from collapsing. BPSC was obtained by the gelation reaction of $\mathrm{TCB}$ and $\mathrm{BTSC}$ according to Equation (1). The structures of TCB, BTSC were investigated by FT-IR spectra as shown in Figure 1, which shows the expected absorption signals. The typical absorption signals of $\mathrm{C}-\mathrm{H}$ (2969, 2941, 2910, $\left.2880 \mathrm{~cm}^{-1}\right), \mathrm{Si}-\mathrm{C}\left(1261,785,745 \mathrm{~cm}^{-1}\right)$ and B-C $\left(1324 \mathrm{~cm}^{-1}\right)$ for TCB were observed. There are also strong absorption signals of $\mathrm{C}-\mathrm{H}\left(2959,2899 \mathrm{~cm}^{-1}\right)$, $\mathrm{N}=\mathrm{C}=\mathrm{N}\left(2176 \mathrm{~cm}^{-1}\right), \mathrm{Si}-\mathrm{C}\left(1251,756 \mathrm{~cm}^{-1}\right)$ and $\mathrm{Si}-\mathrm{C}$ in $\mathrm{Me}_{3} \mathrm{Si}-\left(730 \mathrm{~cm}^{-1}\right)$ for BTSC. 
The expected structures of BPSC were confirmed by its NMR spectra. The ${ }^{29} \mathrm{Si}\left\{{ }^{1} \mathrm{H}\right\} \mathrm{CP}$ MAS NMR resonance signals of $\mathrm{SiC}_{3} \mathrm{~N}$ and $\mathrm{SiC}_{2} \mathrm{~N}_{2}$ were observed at $10.9 \mathrm{ppm}$ and -17.8 ppm, respectively. ${ }^{11} \mathrm{~B}\left\{{ }^{1} \mathrm{H}\right\}$ CP MAS NMR chemical shift assigned to $\mathrm{BC}_{3}$ environment was observed at $3.38 \mathrm{ppm}$. Moreover, the resonance signal of $\mathrm{C}-\mathrm{H}$ in $\mathrm{Me}_{3} \mathrm{SiCl}$ was detected at $0.42 \mathrm{ppm}$ in the liquid ${ }^{1} \mathrm{H}$ NMR spectrum of the soluble fraction of BPSC wet gel. These results indicated that the reaction leads to the formation of a 3D BPSC network fully filled with liquid $\mathrm{Me}_{3} \mathrm{SiCl}$.

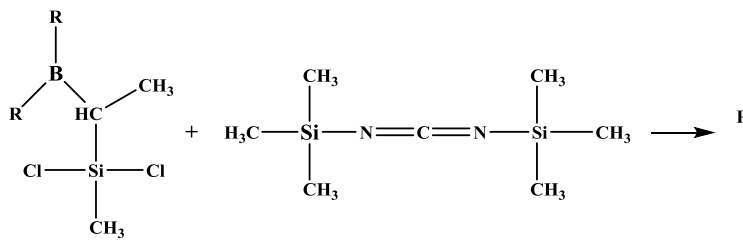

$\mathrm{R}=\mathrm{C}_{2} \mathrm{H}_{4} \mathrm{Si}\left(\mathrm{CH}_{3}\right) \mathrm{Cl}_{2}$

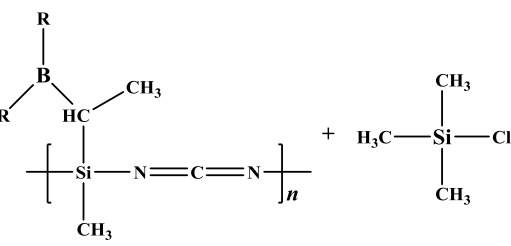

$\mathrm{R}=\left[\mathrm{C}_{2} \mathrm{H}_{4} \mathrm{Si}\left(\mathrm{CH}_{3}\right) \mathrm{NCN}\right]$

According to Equation (1), the liquid $\mathrm{Me}_{3} \mathrm{SiCl}$ is about $65 w t \%$ of the entire wet gel.

If the $\mathrm{Me}_{3} \mathrm{SiCl}$ was directly removed, the 3D BPSC network would collapse due to the surface tension $[6,39,40]$. Herein, we design a novel approach to stabilize the 3D network by replacing $\mathrm{Me}_{3} \mathrm{SiCl}$ with polystyrene (PS) forming a diphasic BPSC-PS xerogel. PS was solid at RT and stable up to $350{ }^{\circ} \mathrm{C}$ (will be discussed in TGA analysis later), which would provide effective support to the 3D BPSC network preventing from collapse. In the FT-IR curve of BPSC-PS (Figure 1), it is observed that there are aliphatic $\mathrm{C}-\mathrm{H}\left(2957,2918 \mathrm{~cm}^{-1}\right), \mathrm{N}=\mathrm{C}=\mathrm{N}\left(2230 \mathrm{~cm}^{-1}\right), \mathrm{Si}-\mathrm{C}\left(1257,760 \mathrm{~cm}^{-1}\right), \mathrm{B}-\mathrm{C}$ $\left(1346 \mathrm{~cm}^{-1}\right)$, phenyl and aromatic C-H for PS (3000 3100, 1601, 1497, $\left.696 \mathrm{~cm}^{-1}\right)$, confirming the formation of PS embedded in BPSC network. The peak corresponding to 
$\mathrm{Me}_{3} \mathrm{Si}-$ end group $\left(730 \mathrm{~cm}^{-1}\right)$ observed in BTSC disappeared in the BPSC-PS xerogel, indicating that the byproduct $\mathrm{Me}_{3} \mathrm{SiCl}$ was replaced by PS completely.

In order to analyze the pyrolysis properties of BPSC-PS xerogel, its TGA curve is shown in Figure 2. The thermal behavior of BPSC-PS is characterized by a three-step mass loss from RT to $950{ }^{\circ} \mathrm{C}$. The first step is the temperature range from RT to $400{ }^{\circ} \mathrm{C}$ with a weight loss of $30 \%$, the second step is from $400{ }^{\circ} \mathrm{C}$ to $480{ }^{\circ} \mathrm{C}$ with an interval weight loss of $50 \%$, and the third step is from $480{ }^{\circ} \mathrm{C}$ to $950{ }^{\circ} \mathrm{C}$ with an interval weight loss of 5\%. This three-step mass loss results a total weight loss of $85 \%$ for BPSC-PS, indicating a total ceramic yield of $15 \%$. This value is equal to the ceramic yield calculated from the differential weight of BPSC-PS and SiBCN-1000. The low ceramic yield indicates that the process is not suitable to produce dense polymer-derived bulk ceramics because it is difficult to inhibit the appearance of cracks [39]. Nevertheless, it is beneficial to produce porous ceramics with the premise that the $3 \mathrm{D}$ porous structure is stable. In order to further analyze the decomposition behaviors, the TGA curves of pure BPSC (after removing $\mathrm{Me}_{3} \mathrm{SiCl}$ ) and PS were obtained and shown in Figure 2. It is observed that these two curves cross at $400{ }^{\circ} \mathrm{C}$ and form a triangle region with the curve of BPSC-PS in the temperature range from $400{ }^{\circ} \mathrm{C}$ to $420{ }^{\circ} \mathrm{C}$. Theoretically, these three TGA curves should intersect at $400{ }^{\circ} \mathrm{C}$. However, the 3D skeleton of BPSC-PS xerogel hinders the decomposing components of PS from running out. As a result, the weight loss rate of PS in the BPSC-PS xerogel is lower than that of pure PS, leading to a 
hysteresis region in the temperature range from $400{ }^{\circ} \mathrm{C}$ to $420{ }^{\circ} \mathrm{C}$. During the BPSC-PS xerogel producing process, the $\mathrm{Me}_{3} \mathrm{SiCl}$ in BPSC wet gel was replaced by styrene and finally formed PS stuffing in the BPSC network. If $\mathrm{Me}_{3} \mathrm{SiCl}(65 w t \%)$ was removed out completely and the same volume of styrene would be introduced into the BPSC wet gel, namely the factions $(f)$ of BPSC and PS in the BPSC-PS xerogel would be $33 w t \%$ and $67 w t \%$, respectively. According to the TGA results, the weight losses $(\Delta)$ of pure BPSC and pure PS are $58 \%$ and $100 \%$, respectively. Thereby, a total weight loss of $86 \%$ is calculated by $f_{\mathrm{BPSC}} \Delta_{\mathrm{BPSC}}+f_{\mathrm{PS}} \Delta_{\mathrm{PS}}$, indicating the final ceramic yield of BPSC-PS xerogel is $14 \%$, which is very close to the ceramic yield from the TGA curve of BPSC-PS (15\%). PS was stable in the temperature range from RT to $350{ }^{\circ} \mathrm{C}$, performing as a support while BPSC cross-linked and transformed into a relative stable 3D skeleton structure. This stable 3D skeleton could avoid the structural collapse even when PS decomposed fast and completely in the temperature range of $350{ }^{\circ} \mathrm{C}$ to $440{ }^{\circ} \mathrm{C}$.

In order to investigate the structural changes during pyrolysis, the FT-IR spectra of BPSC-PS-400 and SiBCN-1000 were measured and shown in Figure 1. There are no peaks for PS found in BPSC-PS-400, indicating the complete removal of PS after pyrolysis at $400{ }^{\circ} \mathrm{C}$ for $4 \mathrm{~h}$. The FT-IR peaks for $-\mathrm{C}=\mathrm{N}-\left(1660 \mathrm{~cm}^{-1}\right)$ observed in BPSC-PS-400 indicates that the Schiff base structure was formed during the pyrolysis, and this structure is originated from the elimination of nitrogen and cyanogen $\left(\mathrm{C}_{2} \mathrm{~N}_{2}\right)$ $[22,39]$. The FT-IR spectrum of SiBCN-1000 shows broad peaks of B-C $\left(1346 \mathrm{~cm}^{-1}\right)$ 
and $\mathrm{Si}-\mathrm{C} / \mathrm{Si}-\mathrm{N}\left(918\right.$ and $807 \mathrm{~cm}^{-1}$ ), which reflect the typical inorganic structures in SiBCN ceramics.

\subsection{Structures of hierarchically porous SiBCN monolith}

Figure 3(a) shows the photograph of BPSC-PS monolith precursor, which was obtained in a cylindrical glass vial with an inner diameter of $1 \mathrm{~cm}$. The BPSC-PS monolith precursor was heated at $400{ }^{\circ} \mathrm{C}$ for $4 \mathrm{~h}$ to completely remove the stuffed PS, following by heating at $1000{ }^{\circ} \mathrm{C}$ for $4 \mathrm{~h}$ under a nitrogen flow to obtain a cylindrical porous SiBCN-1000, as shown in Figure 3(b). It is observed that the SiBCN monolith maintains the cylindrical shape with a diameter of $\sim 0.5 \mathrm{~cm}$.

Figure 4(a) shows the surface morphology of $\mathrm{SiBCN}-1000$. It is observed that SiBCN-1000 contains a large amount of interconnected micron-sized pores. The porous 3D network is continuous and has smooth surface. This mainly attributes to the stable PS which supports the BPSC skeleton when it decomposes in the temperature range from RT to $350{ }^{\circ} \mathrm{C}$. There are also many mesopores $(2 \sim 50 \mathrm{~nm})$ on the backbones of SiBCN-1000 surface as shown in Figure 4(b). Therefore, the architecture of SiBCN-1000 is hierarchically porous with both macropores and mesopores. The macropores $(>50 \mathrm{~nm})$ were originated from the large amount of $\mathrm{Me}_{3} \mathrm{SiCl}(65 w t \%)$ generated in BPSC wet gel. $\mathrm{Me}_{3} \mathrm{SiCl}$ was replaced by PS completely, and then PS decomposed leaving abundant macropores during pyrolysis. The mesopores were formed due to the evaporation of gaseous species during the decomposition of BPSC-PS 
$[6,39]$. The cross-section image of $\mathrm{SiBCN}-1000$ was shown in Figure 4(c). It further confirms that the porous structure in $\mathrm{SiBCN}-1000$ is $3 \mathrm{D}$ continuous. The diameter distribution of macropores was obtained by analyzing the SEM image (Figure 4(a)) using Gatan DigitalMicrograph software and illustrated in Figure 4(d). It is shown that the majority of macropores are in the diameter range of $0.1 \sim 1 \mu \mathrm{m}$ and a few macropores are in the diameter range of $1 \sim 2.3 \mu \mathrm{m}$.

The hierarchically porous architecture of $\mathrm{SiBCN}-1000$ was further characterized by nitrogen adsorption/desorption measurement. Figure 5(a) shows the isotherm curve of SiBCN-1000 which exhibits a typical Type II shape for macroporous materials with a hysteresis in the relative pressure $\left(P / P_{0}\right)$ range of $0.8 \sim 1.0$. The pore volume $\left(V_{\mathrm{g}}\right)$ is $0.335 \mathrm{~cm}^{3} \cdot \mathrm{g}^{-1}$, which was estimated from the amount adsorbed at a relative pressure of 0.99 from the isotherm curve. The density of $\operatorname{SiBCN}-1000(\rho)$ is $1.50 \mathrm{~g} \cdot \mathrm{cm}^{-3}$ obtained by Archimedes' method, corresponding to a porosity $(P)$ of 50.4 vol\% $\left(P=\rho V_{\mathrm{g}}\right)$. The specific surface area is $31.78 \mathrm{~m}^{2} \cdot \mathrm{g}^{-1}$ calculated by the Brunauer-Emmett-Teller (BET) method. The pore volume is close to the results $\left(0.25\right.$ to $\left.0.35 \mathrm{~cm}^{3} \cdot \mathrm{g}^{-1}\right)$ of hierarchically porous SiBCN monoliths prepared by SPS, while the specific surface area is relatively smaller than that [4]. This is due to the highly interconnected macropores in SiBCN-1000 leading to high pore volume but low surface area, while more mesopores deducing high surface area as reported in the reference [4]. The pore-size distribution curve of $\mathrm{SiBCN}-1000$ monolith was calculated from the desorption branch of its 
isotherm curve by Barrett-Joyner-Halenda $(\mathrm{BJH})$ method and shown in Figure 5(b). It is observed that SiBCN-1000 monolith contains a major amount of micro- and mesopores with a size range of $1 \sim 10 \mathrm{~nm}$ and has an average pore diameter of $1.95 \mathrm{~nm}$. The formation of micropores $(<2 \mathrm{~nm})$ is due to the liberation of gaseous species during the decomposition of BPSC-PS. The broad huddle between $10 \sim 174.5 \mathrm{~nm}$ in the BJH curve represents the mesopores and macropores observed in the SEM images (Figure 4). Thereby, the hierarchically porous $\mathrm{SiBCN}$ monolith in which the micropores and mesopores are interconnected with a continuous macroporous network was obtained.

\subsection{High temperature stability of porous $\mathrm{SiBCN}$ monolith}

To analyze the high temperature stability, SiBCN-1000 was further annealed at 1500 ${ }^{\circ} \mathrm{C}$ for $4 \mathrm{~h}$ under a nitrogen flow and the obtained SiBCN monolith was labeled as SiBCN-1500. The surface SEM image of SiBCN-1500 was shown in Figure 6(a). It displays a similar morphology as $\mathrm{SiBCN}-1000$, but with a smaller micron-size pore diameter and narrower pore distribution. The partial enlarged surface SEM image (Figure 6(b)) shows distinct interconnected macropores throughout the monolith and many mesopores distributing in the backbones. These results indicate that $\mathrm{SiBCN}-1500$ maintains the hierarchically porous structure but has different pore size and distribution compared to SiBCN-1000.

In order to further analyze the differences of the hierarchically porous architectures between SiBCN-1500 and SiBCN-1000, the nitrogen adsorption/desorption isotherm of 
SiBCN-1500 was obtained and shown in Figure 7(a). This isotherm curve exhibits a typical Type II shape same as that for SiBCN-1000, while a distinct hysteresis in the relative pressure range of $0.4 \sim 1.0$ is observed, suggesting that $\mathrm{SiBCN}-1500$ contains much more mesopores than $\mathrm{SiBCN}-1000$. The pore volume of $\mathrm{SiBCN}-1500$ is 0.201 $\mathrm{cm}^{3} \cdot \mathrm{g}^{-1}$, less than $0.335 \mathrm{~cm}^{3} \cdot \mathrm{g}^{-1}$ of $\mathrm{SiBCN}-1000$, implying that the amount of macropores decreased after annealing at $1500{ }^{\circ} \mathrm{C}$. The specific surface area of SiBCN-1500 calculated by BET method is $44.60 \mathrm{~m}^{2} \cdot \mathrm{g}^{-1}$, larger than $31.78 \mathrm{~m}^{2} \cdot \mathrm{g}^{-1}$ for SiBCN-1000, confirming that there are more mesopores formed in SiBCN-1500. The pore distribution of SiBCN-1500 calculated by BJH method is shown in Figure 7(b). It is observed that the pore diameter is in the range of $3.06 \sim 103.23 \mathrm{~nm}$ with an average pore diameter of $3.81 \mathrm{~nm}$. The majority of pores have a diameter range of 3.06 to $30 \mathrm{~nm}$. No micropores $(<2 \mathrm{~nm})$ were detected in the pore distribution curve. The disappearance of micropores might be due to the carbothermal reduction reaction occurred during the high temperature annealing $\left(>1440{ }^{\circ} \mathrm{C}\right)[39]$, which consumed the free $\mathrm{C}$ in the pore walls, leading to the enlargement of micropores to mesopores. This result explains the increasing amount of mesopores as well. Combining with the SEM results (smaller micron-size pore diameter and narrower pore distribution of SiBCN-1500 than SiBCN-1000), it can conclude that a pore size homogenization process occurred during the high temperature annealing, due to which more mesopores $(2 \sim 50 \mathrm{~nm})$ introduced in $\mathrm{SiBCN}-1500$ along with the disappearance of micropores $(<2 \mathrm{~nm})$ and the reduction 
of macropores $(>50 \mathrm{~nm})$. This process results in the shrinkage of SiBCN-1000. The volume shrinking percentage $(S)$ is about $21 \%$ calculated by $S=$ $\left(V_{\mathrm{SiBCN}-1000}-V_{\mathrm{SiBCN}-1500}\right) \times 100 \% / V_{\mathrm{SiBCN}-1000}$, while $V_{\mathrm{SiBCN}-1000}$ and $V_{\mathrm{SiBCN}-1500}$ are the volumes of $\mathrm{SiBCN}-1000$ and $\mathrm{SiBCN}-1500$, respectively.

The XRD patterns of SiBCN-1000 and SiBCN-1500 are shown in Figure 8(a) to investigate the mechanism of the pore size homogenization process during the high temperature annealing. It is observed that both $\mathrm{SiBCN}-1000$ and $\mathrm{SiBCN}-1500$ present typical amorphous characteristics with three dispersing diffraction peaks, confirming that the SiBCN-1500 maintains the amorphous skeleton structure of SiBCN-1000. The first principal dispersing diffraction peak of XRD pattern has been widely used to analyze the medium-range local ordering structures in amorphous materials [41-43]. It is observed that the first principal diffraction peak of XRD pattern for SiBCN-1500 is sharper than that for $\mathrm{SiBCN}-1000$, indicating that there are more uniform local-ordering structures in $\mathrm{SiBCN}-1500$. In order to obtain the medium-range ordering distances in SiBCN-1000 and SiBCN-1500, a Lorentz method was used to fit the XRD curves in Figure 8(a) and the obtained fitting curves are shown in Figure 8(b) and (c). The $d_{\text {Brag }}$ spacings of the first principal dispersing diffraction peaks are $0.44 \mathrm{~nm}$ and $0.40 \mathrm{~nm}$ calculated by Bragg equation for $\mathrm{SiBCN}-1000$ and $\mathrm{SiBCN}-1500$, respectively. For amorphous ceramics, $d_{\text {Brag }}$ should be corrected with a factor of 1.22 to obtain the average medium-range ordering distance $\left(r^{\mathrm{M}}\right)$ derived by two adjacent particles [41-43], 
namely the $r^{\mathrm{M}}$ values are $0.54 \mathrm{~nm}$ and $0.49 \mathrm{~nm}$ for SiBCN-1000 and SiBCN-1500, respectively. The smaller medium-range local ordering distance in SiBCN-1500 may be due to the carbothermal reduction reaction occurring above $1440{ }^{\circ} \mathrm{C}[39,44]$, somewhat destroyed the original local-ordering structures in SiBCN-1000.

Therefore, the mechanism of the pore size homogenization process for hierarchically porous $\mathrm{SiBCN}$ monolith annealing at $1500{ }^{\circ} \mathrm{C}$ could be proposed as follows. For macropores, which are mainly the voids among the SiBCN skeletons (Figure 4(c)), a process similar to powder sintering densification happens during the annealing of SiBCN-1000 at $1500{ }^{\circ} \mathrm{C}$, leading to the reduction of macropores $(>50 \mathrm{~nm})$ in $\mathrm{SiBCN}-1500$. In this process, the $\mathrm{SiBCN}$ skeletons separated by macropores are analogized as particles, and the free $\mathrm{C}$ and $\mathrm{B}$ act as sintering aid. While for micropores and mesopores, which are mainly in the skeleton (Figure 4(c)), a pore enlargement process occurs due to the carbothermal reduction reaction, which consumes the free $\mathrm{C}$ in the pore walls and enlarges the micropores and mesopores, leading to the disappearance of micropores and a larger average pore size in SiBCN-1500. These two processes generate more mesopores in SiBCN-1500.

\section{Conclusion}

The template-free synthesis method for the hierarchically porous SiBCN monolith has been established by in-situ pyrolyzing organic gel precursors. The key process of this method is the complete replacement of $\mathrm{Me}_{3} \mathrm{SiCl}$ by PS, which stuffs the pores and 
stabilizes the 3D BPSC network skeleton during the pyrolysis. The obtained hierarchically porous $\mathrm{SiBCN}$ monolith has a cylindrical shape, in which the micropores and mesopores are interconnected with a continuous macroporous network. The high temperature stability study indicates that the hierarchically porous structures with amorphous skeletons could be maintained even when the monolith is annealed at 1500 ${ }^{\circ} \mathrm{C}$. Due to the simple fabrication process of organic gel precursors, this method can be used to prepare various hierarchically porous non-oxide monoliths.

\section{- AUTHOR INFORMATION}

\section{Corresponding Author}

*E-mail: linan.an@ucf.edu (L.A); wangca@tsinghua.edu.cn (C.W)

\section{- ACKNOWLEDGMENT}

The work is financially supported by Department of Energy of USA (DE-FE0007004).

L.Y. would like to acknowledge financial support from China Scholarship Council.

\section{- REFERENCES}

[1] N.D. Petkovich, A. Stein, Controlling macro- and mesostructures with hierarchical porosity through combined hard and soft templating, Chem Soc Rev, 42 (2013) 3721-3739.

[2] Y. Li, Z.Y. Fu, B.L. Su, Hierarchically Structured Porous Materials for Energy Conversion and Storage, Adv Funct Mater, 22 (2012) 4634-4667. 
[3] X. Li, M. Sun, J.C. Rooke, L. Chen, B.-L. Su, Synthesis and applications of hierarchically porous catalysts, Chinese J Catal, 34 (2013) 22-47.

[4] O. Majoulet, F. Sandra, M.C. Bechelany, G. Bonnefont, G. Fantozzi, L. Joly-Pottuz, A. Malchere, S. Bernard, P. Miele, Silicon-boron-carbon-nitrogen monoliths with high, interconnected and hierarchical porosity, J Mater Chem A, 1 (2013) 10991-11000.

[5] I.K. Sung, Christian, M. Mitchell, D.P. Kim, P.J.A. Kenis, Tailored macroporous $\mathrm{SiCN}$ and $\mathrm{SiC}$ structures for high-temperature fuel reforming, Adv Funct Mater, 15 (2005) 1336-1342.

[6] A.O. Gabriel, R. Riedel, Preparation of non-oxidic silicon ceramics by an anhydrous sol-gel process, Angew Chem Int Edit, 36 (1997) 384-386.

[7] K. Lippe, J. Wagler, E. Kroke, S. Herkenhoff, V. Ischenko, J. Woltersdorf, Cyclic Silylcarbodiimides as Precursors for Porous Si/C/N Materials: Formation, Structures, and Stabilities, Chem Mater, 21 (2009) 3941-3949.

[8] S. Bernard, P. Miele, Ordered mesoporous polymer-derived ceramics and their processing into hierarchically porous boron nitride and silicoboron carbonitride monoliths, New J Chem, 38 (2014) 1923-1931.

[9] A.R. Passos, L. Martins, S.H. Pulcinelli, C.V. Santilli, Design of hierarchical porous aluminas by using one-pot synthesis and different calcination temperatures, J Sol-Gel Sci Techn, 63 (2012) 242-250.

[10] E. Ortel, S. Sokolov, C. Zielke, I. Lauermann, S. Selve, K. Weh, B. Paul, J. Polte, R. Kraehnert, Supported Mesoporous and Hierarchical Porous Pd/TiO2 Catalytic Coatings with Controlled Particle Size and Pore Structure, Chem Mater, 24 (2012) 3828-3838.

[11] J.G. Alauzun, S. Ungureanu, N. Brun, S. Bernard, P. Miele, R. Backov, C. Sanchez, Novel monolith-type boron nitride hierarchical foams obtained through integrative chemistry, J Mater Chem, 21 (2011) 14025-14030.

[12] M. Kamperman, A. Burns, R. Weissgraeber, N. van Vegten, S.C. Warren, S.M. Gruner, A. Baiker, U. Wiesner, Integrating Structure Control over Multiple Length Scales in Porous High Temperature Ceramics with Functional Platinum Nanoparticles, Nano Lett, 9 (2009) 2756-2762.

[13] K. Sonnenburg, P. Adelhelm, M. Antonietti, B. Smarsly, R. Noske, P. Strauch, Synthesis and characterization of $\mathrm{SiC}$ materials with hierarchical porosity obtained by replication techniques, Phys Chem Chem Phys, 8 (2006) 3561-3566.

[14] H. Wang, X.D. Li, J.S. Yu, D.P. Kim, Fabrication and characterization of ordered macroporous PMS-derived SiC from a sacrificial template method, J Mater Chem, 14 (2004) 1383-1386.

[15] C. Vakifahmetoglu, I. Menapace, A. Hirsch, L. Biasetto, R. Hauser, R. Riedel, P. Colombo, Highly porous macro- and micro-cellular ceramics from a polysilazane precursor, Ceram Int, 35 (2009) 3281-3290.

[16] M. Fukushima, P. Colombo, Silicon carbide-based foams from direct blowing of polycarbosilane, J Eur Ceram Soc, 32 (2012) 503-510. 
[17] B.H. Yoon, E.J. Lee, H.E. Kim, Y.H. Koh, Highly aligned porous silicon carbide ceramics by freezing polycarbosilane/camphene solution, J Am Ceram Soc, 90 (2007) 1753-1759.

[18] P. Colombo, G. Mera, R. Riedel, G.D. Soraru, Polymer-Derived Ceramics: 40 Years of Research and Innovation in Advanced Ceramics, J Am Ceram Soc, 93 (2010) $1805-1837$.

[19] X.B. Yan, L. Gottardo, S. Bernard, P. Dibandjo, A. Brioude, H. Moutaabbid, P. Miele, Ordered Mesoporous Silicoboron Carbonitride Materials via Preceramic Polymer Nanocasting, Chem Mater, 20 (2008) 6325-6334.

[20] O. Majoulet, J.G. Alauzun, L. Gottardo, C. Gervais, M.E. Schuster, S. Bernard, P. Miele, Ordered mesoporous silicoboron carbonitride ceramics from boron-modified polysilazanes: Polymer synthesis, processing and properties, Micropor Mesopor Mat, 140 (2011) 40-50.

[21] R. Riedel, A. Kienzle, W. Dressler, L. Ruwisch, J. Bill, F. Aldinger, A silicoboron carbonitride ceramic stable to $2,000{ }^{\circ} \mathrm{C}$, Nature, 382 (1996) 796-798.

[22] M. Weinmann, R. Haug, J. Bill, F. Aldinger, J. Schuhmacher, K. Muller, Boron-containing polysilylcarbodi-imides: a new class of molecular precursors for Si-B-C-N ceramics, J Organomet Chem, 541 (1997) 345-353.

[23] P. Baldus, M. Jansen, D. Sporn, Ceramic fibers for matrix composites in high-temperature engine applications, Science, 285 (1999) 699-703.

[24] M. Weinmann, J. Schuhmacher, H. Kummer, S. Prinz, J.Q. Peng, H.J. Seifert, M. Christ, K. Muller, J. Bill, F. Aldinger, Synthesis and thermal behavior of novel Si-B-C-N ceramic precursors, Chem Mater, 12 (2000) 623-632.

[25] Z.-C. Wang, F. Aldinger, R. Riedel, Novel Silicon-Boron-Carbon-Nitrogen Materials Thermally Stable up to $2200{ }^{\circ} \mathrm{C}$, J Am Ceram Soc, 84 (2001) 2179-2183.

[26] M. Weinmann, T.W. Kamphowe, J. Schuhmacher, K. Muller, F. Aldinger, Design of polymeric Si-B-C-N ceramic precursors for application in fiber-reinforced composite materials, Chem Mater, 12 (2000) 2112-2122.

[27] S. Bernard, M. Weinmann, P. Gerstel, P. Miele, F. Aldinger, Boron-modified polysilazane as a novel single-source precursor for SiBCN ceramic fibers: synthesis, melt-spinning, curing and ceramic conversion, J Mater Chem, 15 (2005) 289-299.

[28] L. Gottardo, S. Bernard, C. Gervais, M. Weinmann, P. Miele, Study of the intermediate pyrolysis steps and mechanism identification of polymer-derived SiBCN ceramics, J Mater Chem, 22 (2012) 17923-17933.

[29] L.G. Zhang, Y.S. Wang, Y. Wei, W.X. Xu, D.J. Fang, L. Zhai, K.C. Lin, L.N. An, A silicon carbonitride ceramic with anomalously high piezoresistivity, J Am Ceram Soc, 91 (2008) 1346-1349.

[30] Y.S. Wang, L.G. Zhang, W.X. Xu, T. Jiang, Y. Fan, D.P. Jiang, L.N. An, Effect of Thermal Initiator Concentration on the Electrical Behavior of Polymer-Derived Amorphous Silicon Carbonitrides, J Am Ceram Soc, 91 (2008) 3971-3975. 
[31] H.Y. Ryu, Q. Wang, R. Raj, Ultrahigh-Temperature Semiconductors Made from Polymer-Derived Ceramics, J Am Ceram Soc, 93 (2010) 1668-1676.

[32] Y.H. Chen, F.Q. Yang, L.N. An, On electric conduction of amorphous silicon carbonitride derived from a polymeric precursor, Appl Phys Lett, 102 (2013) 2163-2167.

[33] L. An, W. Xu, S. Rajagopalan, C. Wang, H. Wang, Y. Fan, L. Zhang, D. Jiang, J. Kapat, L. Chow, B. Guo, J. Liang, R. Vaidyanathan, Carbon-Nanotube-Reinforced Polymer-Derived Ceramic Composites, Adv Mater, 16 (2004) 2036-2040.

[34] H.X. Duan, C. Li, W.W. Yang, B. Lojewski, L.N. An, W.W. Deng, Near-Field Electrospray Microprinting of Polymer-Derived Ceramics, J Microelectromech S, 22 (2013) 1-3.

[35] L.A. Liew, W.G. Zhang, L.N. An, S. Shah, R.L. Luo, Y.P. Liu, T. Cross, M.L. Dunn, V. Bright, J.W. Daily, R. Raj, K. Anseth, Ceramic MEMS - New materials, innovative processing and future applications, Am Ceram Soc Bull, 80 (2001) 25-30.

[36] S. Sarkar, A. Chunder, W.F. Fei, L.N. An, L. Zhai, Superhydrophobic mats of polymer-derived ceramic fibers, J Am Ceram Soc, 91 (2008) 2751-2755.

[37] S. Sarkar, J.H. Zou, J.H. Liu, C.Y. Xu, L.A. An, L. Zhai, Polymer-Derived Ceramic Composite Fibers with Aligned Pristine Multiwalled Carbon Nanotubes, Acs Appl Mater Inter, 2 (2010) 1150-1156.

[38] X.P. Yang, Y.H. Chen, Y.J. Cao, L.N. An, Silicon Carbonitride Hollow Nanospheres from a Block-Copolymer Precursor, J Am Ceram Soc, 97 (2014) 2387-2389.

[39] M. Weinmann, R. Haug, J. Bill, M. de Guire, F. Aldinger, Boron-modified polysilylcarbodi-imides as precursors for Si-B-C-N ceramics: Synthesis, plastic-forming and high-temperature behavior, Appl Organomet Chem, 12 (1998) 725-734.

[40] N. Husing, U. Schubert, Aerogels airy materials: Chemistry, structure, and properties, Angew Chem Int Edit, 37 (1998) 23-45.

[41] L.E.A. Harold P. Klug, X-Ray Diffraction Procedures: For Polycrystalline and Amorphous Materials, John Wiley \& Sons, Inc.1974.

[42] L.E. Alexander, X-ray Diffraction Methods in Polymer Science, John Wiley \& Sons, Inc. 1969.

[43] S. Krimm, A.V. Tobolsky, Quantitative X-Ray Studies of Order in Amorphous and Crystalline Polymers: Scattering from Various Polymers and a Study of the Glass Transition in Polystyrene and Polymethyl Methacrylate, Text Res J, 21 (1951) 805-822. [44] R.M. Prasad, G. Mera, K. Morita, M. Müller, H.-J. Kleebe, A. Gurlo, C. Fasel, R. Riedel, Thermal decomposition of carbon-rich polymer-derived silicon carbonitrides leading to ceramics with high specific surface area and tunable micro- and mesoporosity, J Eur Ceram Soc, 32 (2012) 477-484. 
Figure 1. FT-IR spectra of BTSC, TCB, BPSC-PS xerogel, BPSC-PS-400 and SiBCN-1000. 
Figure 2. TGA curves of pure BPSC (after removing $\mathrm{Me}_{3} \mathrm{SiCl}$ ), PS and BPSC-PS. 
Figure 3. Photographs of (a) BPSC-PS monolith and (b) SiBCN-1000. 
Figure 4. (a) Surface SEM image of SiBCN-1000; (b) Partial enlarged image of Figure 4(a); (c) Cross-section SEM image of SiBCN-1000; (d) Diameter distribution of macropores measured from SEM images (Figure 4(a)). 
Figure 5. (a) Nitrogen adsorption/desorption isotherm of SiBCN-1000; (b) Pore size distribution curve calculated by BJH method from the isotherm curve of SiBCN-1000. 
Figure 6. (a) SEM image of SiBCN-1500; (b) Partial enlarged image of Figure 6(a). 
Figure 7. (a) Nitrogen adsorption/desorption isotherm of SiBCN-1500; (b) Pore size distribution calculated by BJH method from the isotherm curve shown in Figure 7(a). 
Figure 8. (a) XRD curves of SiBCN-1000 and SiBCN-1500; (b) Fitting curves of XRD data for SiBCN-1000; (C) Fitting curves of XRD data for SiBCN-1500. 


\title{
SiBCN-1000
}

\section{BPSC-PS-400}

BPSC-PS
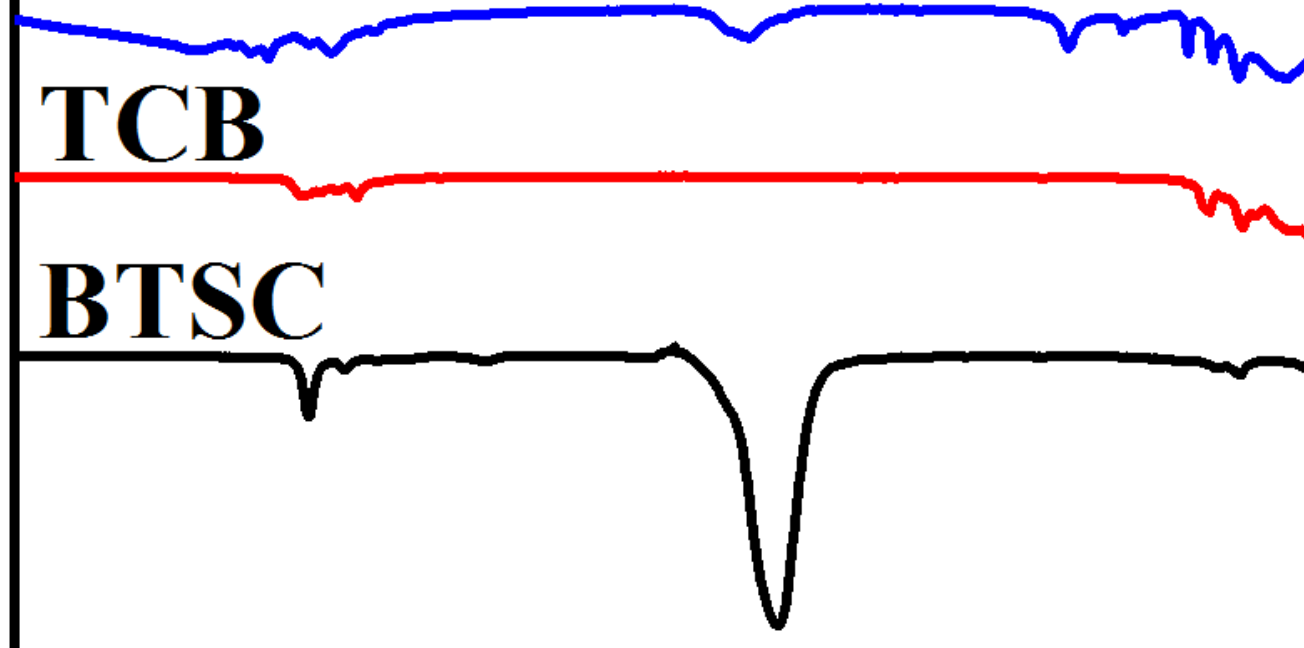

3000

\author{
Wavenumbers $/ \mathrm{cm}^{-1}$
}




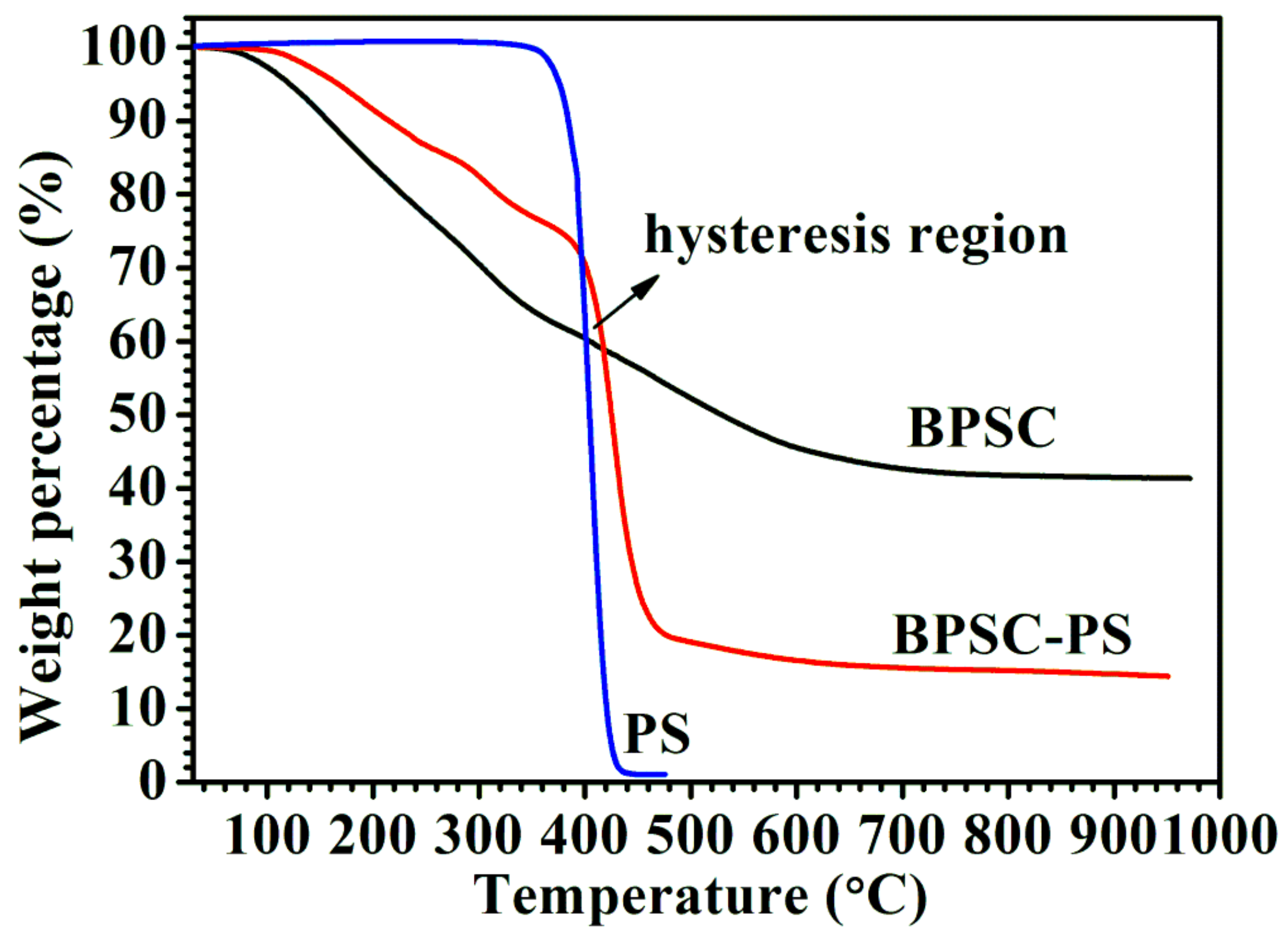


Figure 3

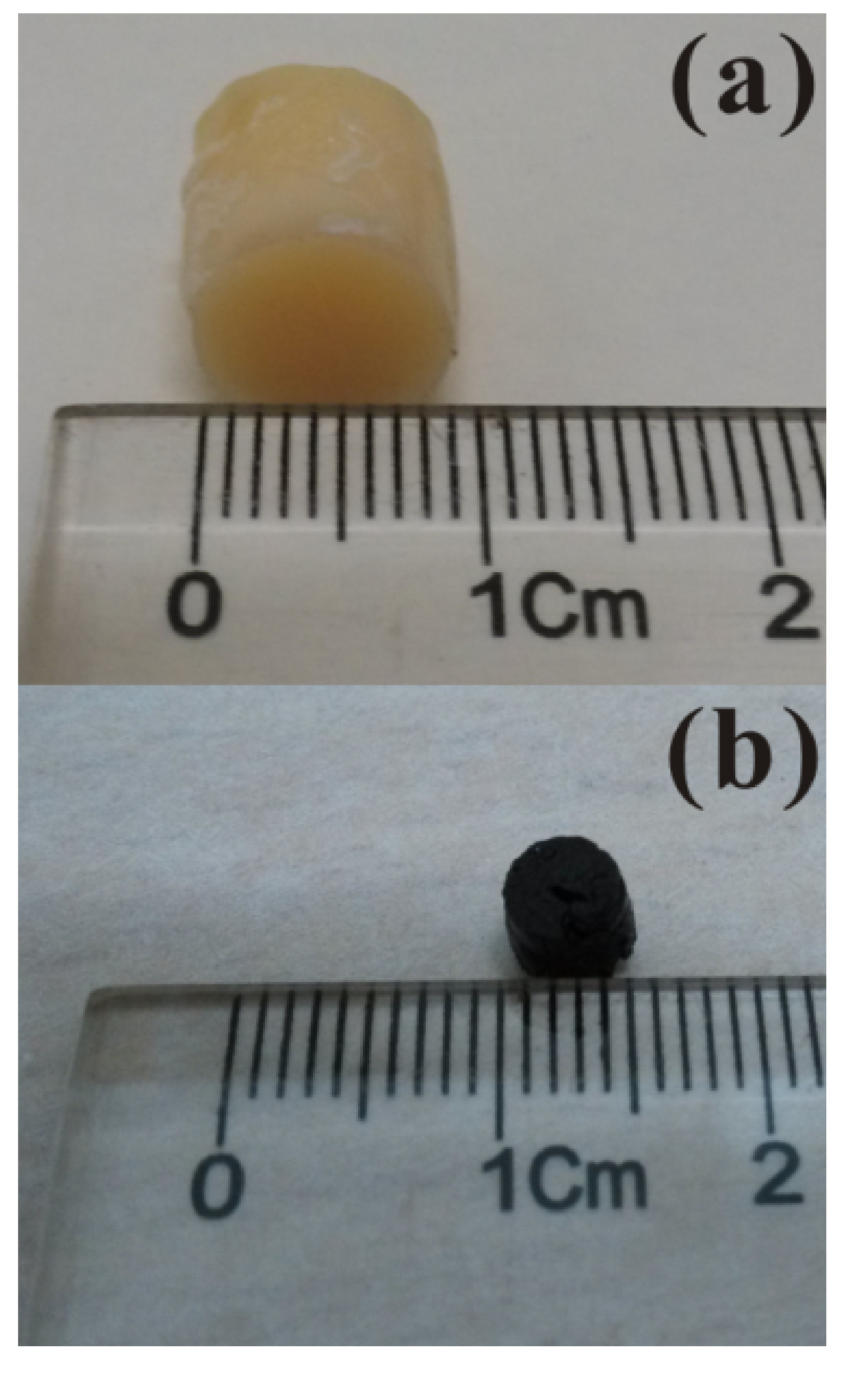

\section{gure 3}

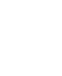

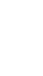
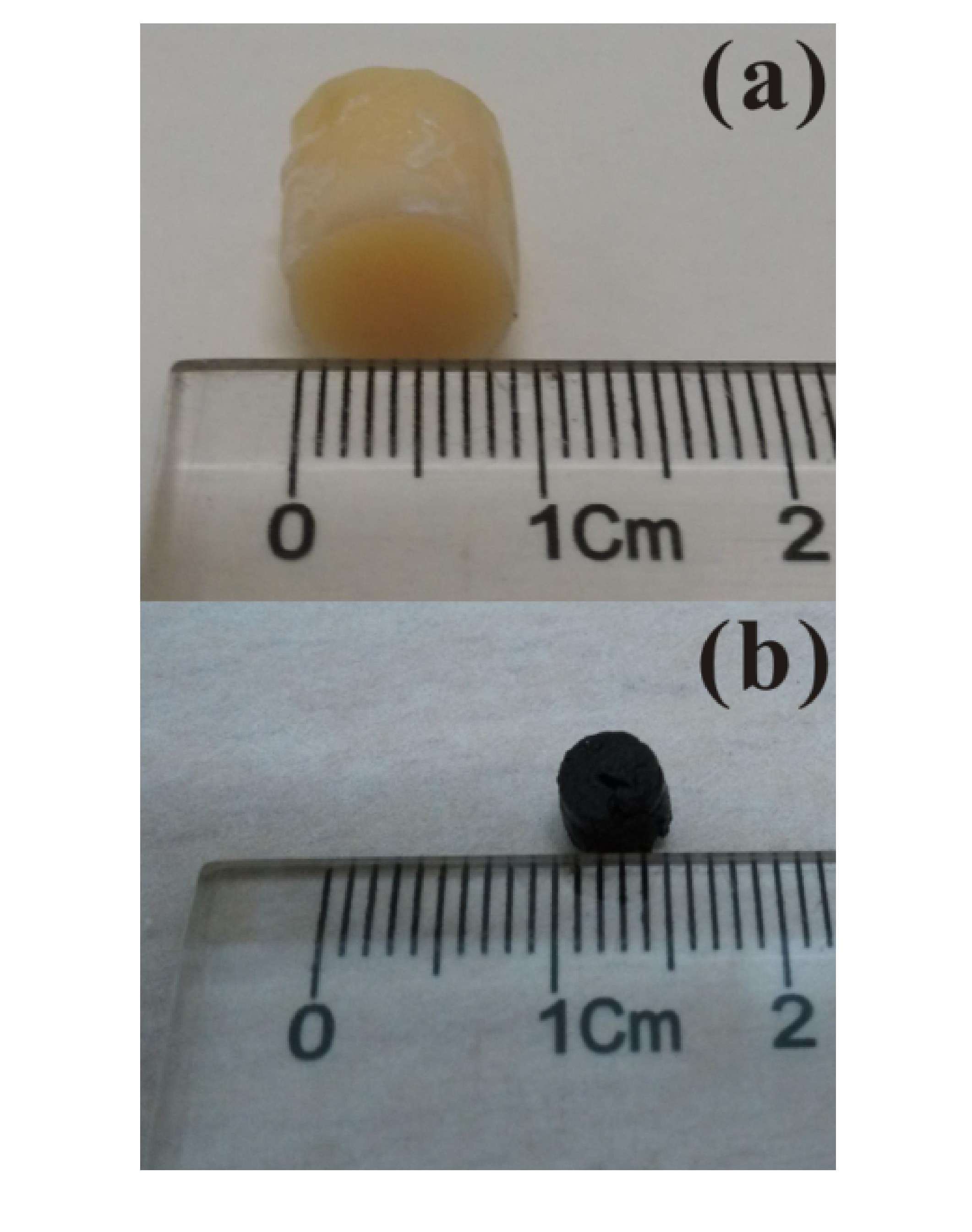

列



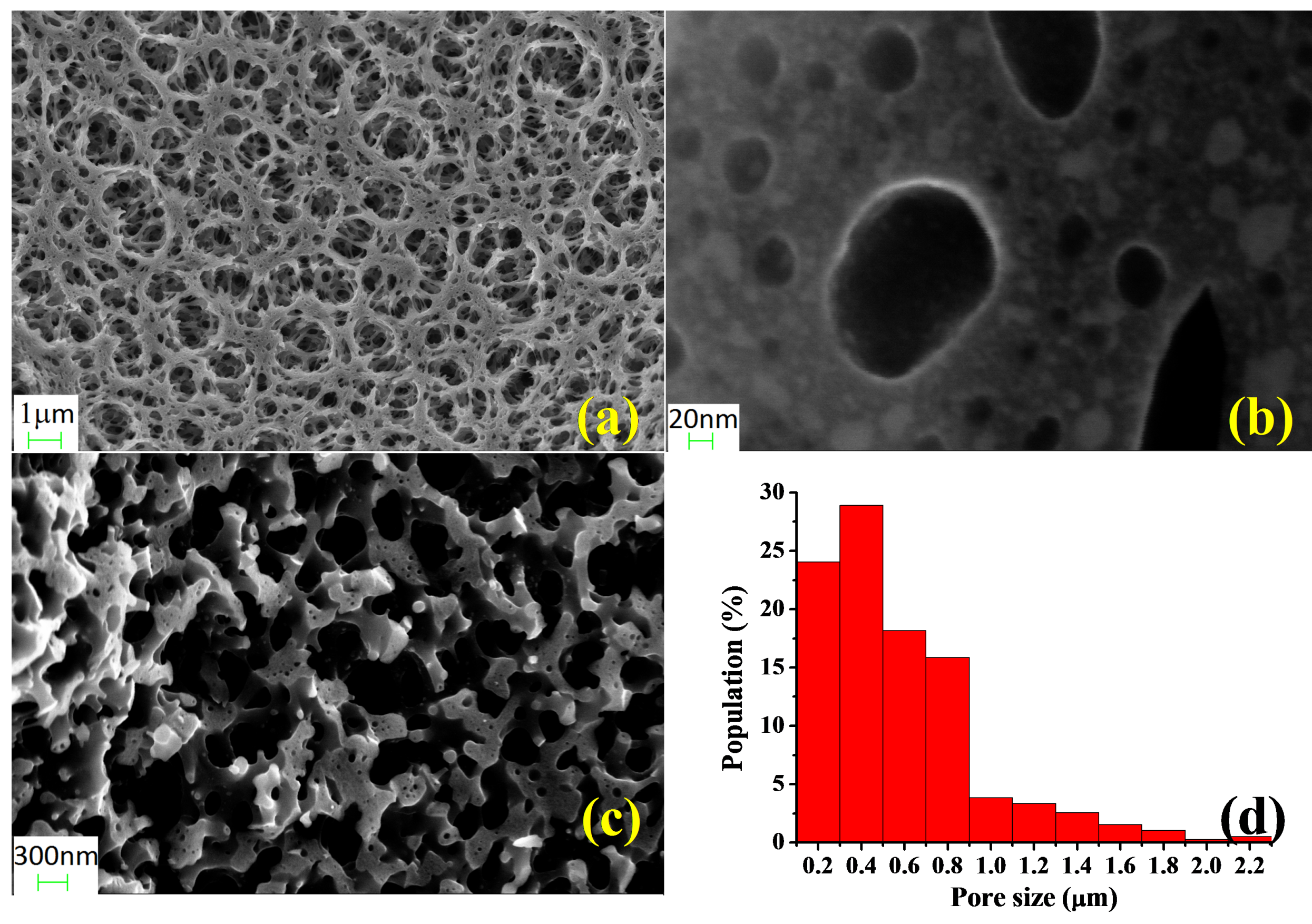

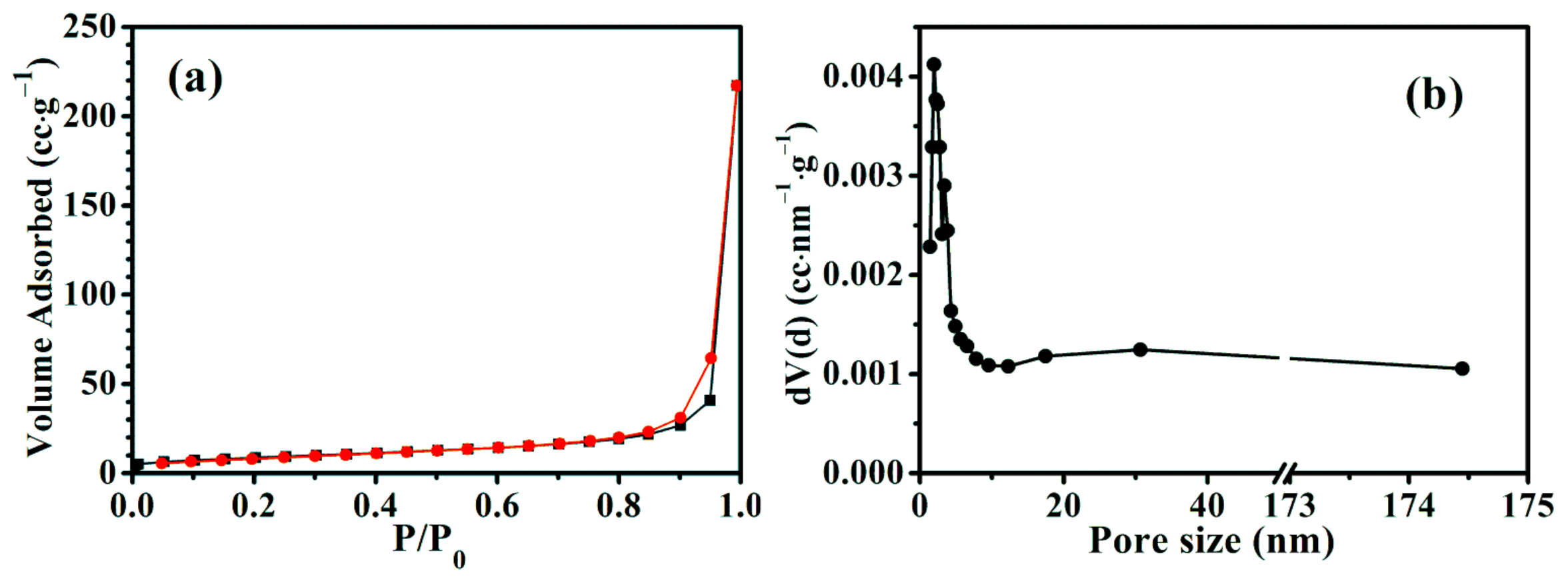

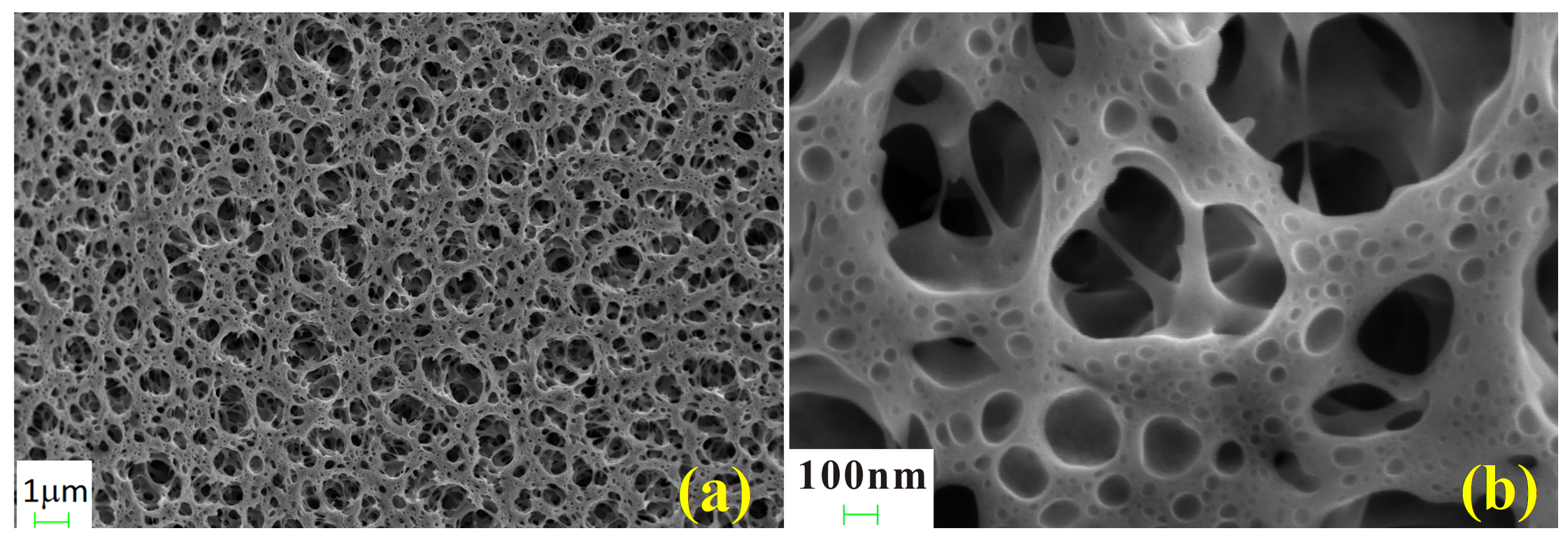

$\longmapsto$ 

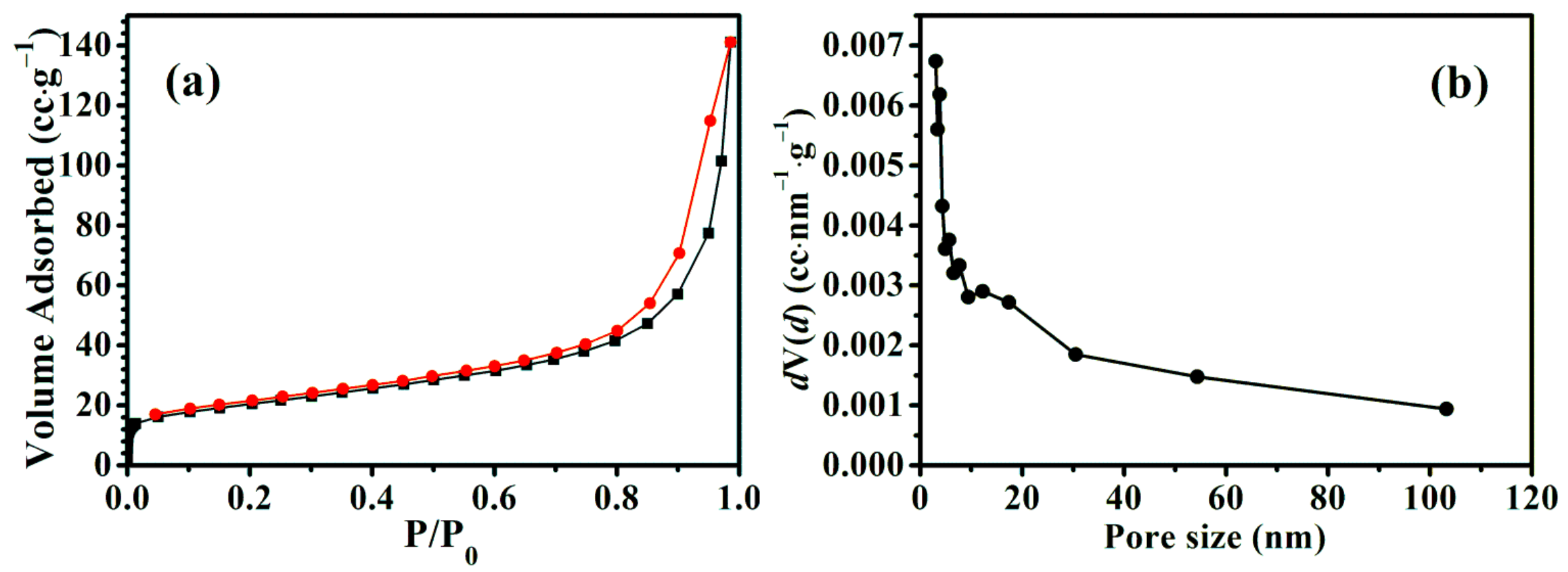

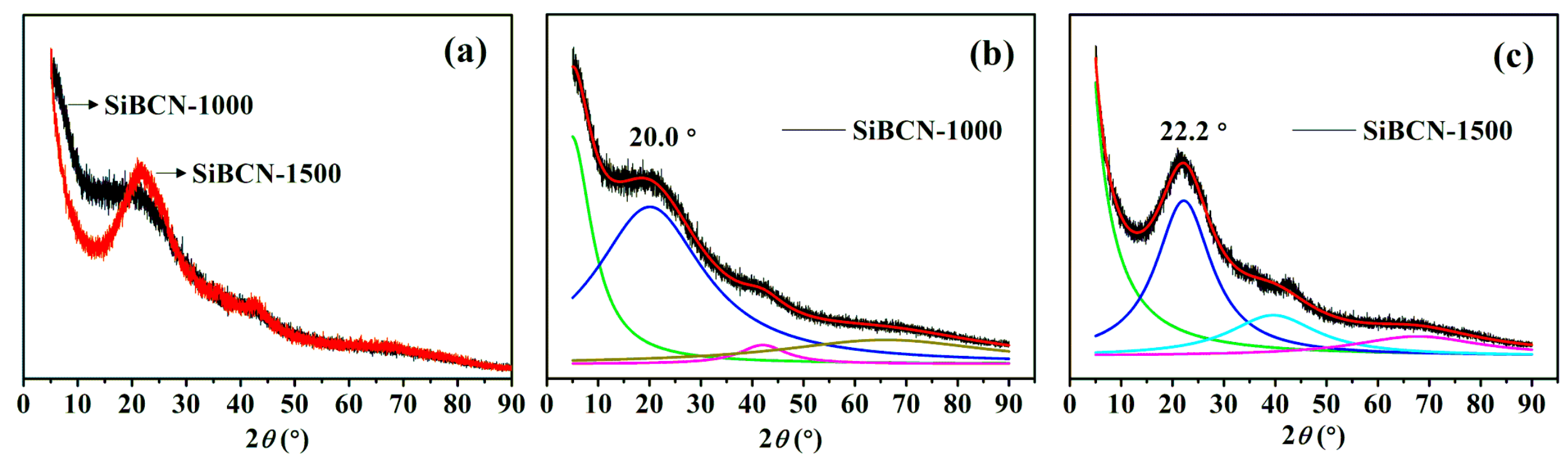